\title{
Acrometastasis from a Pancreatic Primary Adenocarcinoma: A First Report in
} the Literature

\section{O’Grady J*, Collins D, O'Reilly S and Power DG}

Department of Medical Oncology, Cork University Hospital, Wilton, Cork, Ireland

${ }^{*}$ Corresponding author: O'Grady J, Department of Medical Oncology, Cork University Hospital, Wilton, Cork, Ireland, Tel: 00353861672725, E-mail: jogrady@live.ie

Citation: O'Grady J, Collins D, O’Reilly S, Power DG (2014) Acrometastasis from a Pancreatic Primary Adenocarcinoma: A First Report in the Literature. J Case Rep Stud 2(6): 605. doi: 10.15744/2348-9820.2.305

Received Date: October 27, 2014 Accepted Date: December 03, 2014 Published Date: December 05, 2014

\begin{abstract}
A seventy five year male presented with acute onset right foot pain and swelling. Plain imaging revealed a lytic area in the medial and middle cuneiform bones of the right foot. Background history of resected pancreatic cancer and current presentation were consistent early diffuse disease recurrence of a pancreatic primary tumour. The presenting feature in this case was of symptomatic acrometastases, that is, metastases to the feet. Acrometastases have been described in association with multiple primary tumours, but not pancreatic cancer. Our case highlights rare sites of metastatic disease secondary to pancreatic cancer, and we review the literature.
\end{abstract}

\section{Case report}

A seventy five year old retired business man presented with acute onset right foot pain and swelling. He was unable to weight bear due to the severity of symptoms. There was no history of trauma or rheumatologic disorder. Following initial review, he underwent plain imaging of his right foot. This revealed a lytic area in the medial and middle cuneiform bones of the right foot and suspected involvement of the lateral cuneiform bone (Figure 1).

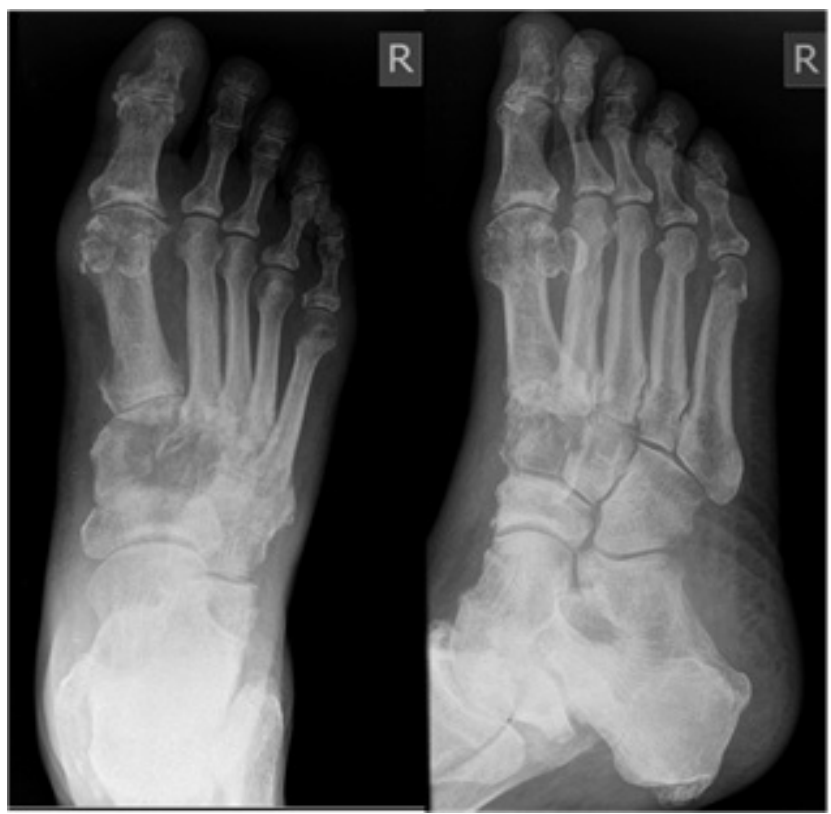

Figure 1: Lytic lesion on plain imaging

Background history was significant for pancreatic adenocarcinoma. He had undergone surgical resection of the pancreatic tumour (Whipple's procedure) ten weeks prior to this current presentation which also included excision of a solitary liver metastasis, which had been an unexpected intraoperative finding. He was due to receive gemcitabine chemotherapy following multidisciplinary team discussion postoperatively, but presented acutely prior to this with his current complaint. Tumour marker analysis on this admission revealed a CA 19-9 level of $1489 \mathrm{kU} / \mathrm{L}$ (normal range 0-37 kU/L). Preoperative CA 19-9 was $934 \mathrm{kU} / \mathrm{L}$ three months prior to this.

A Technetium- 99 Isotope bone scan revealed significantly increased tracer uptake in the right tarsal bones and the right first tarsometatarsal joint (Figure 2), consistent with the acute presenting feature. Increased tracer uptake, with less avidity, was also noted near the proximal right tibia, left tarsal joints and left ankle joint. The areas of most avid activity corresponded with the patient's symptom site as well as the lytic lesions described on plain imaging. All findings were consistent with bone metastases. 


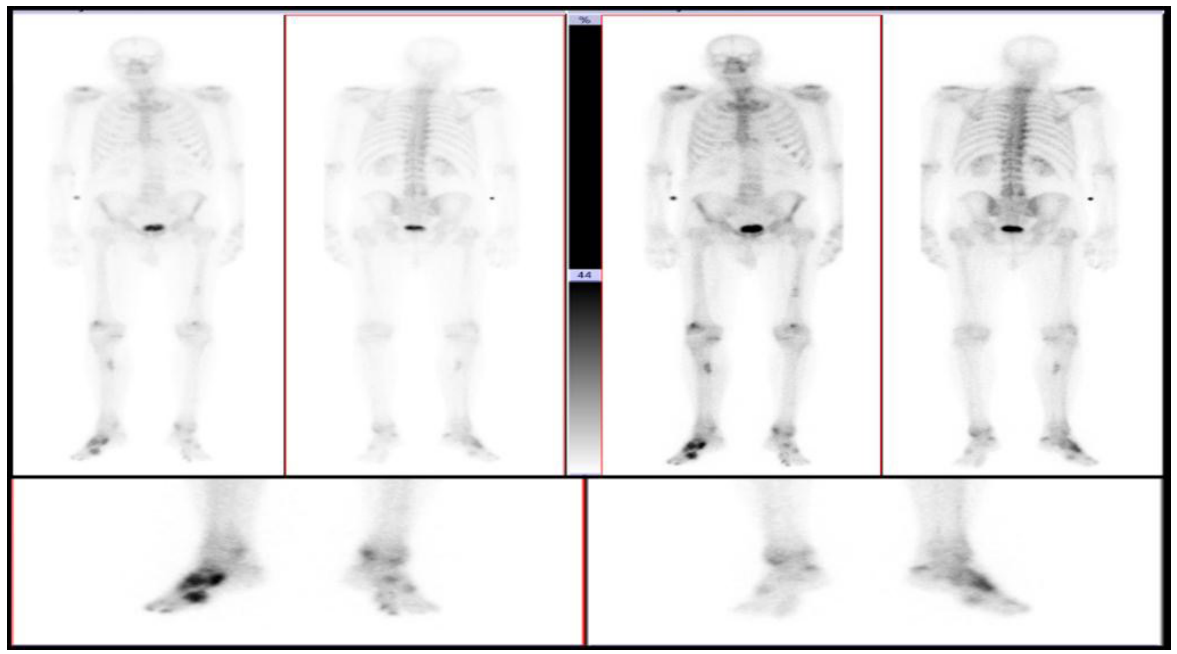

Figure 2: Whole Body Bone Scan with areas of increased tracer uptake

Further cross sectional computed tomography imaging of the thorax, abdomen and pelvis revealed multiple new pulmonary nodules and a soft tissue mediastinal mass, partially encasing the descending thoracic aorta (Figure 3). Within the abdomen, multiple new hepatic lesions, throughout both lobes, were visualized. All findings were consistent with metastatic disease. Multiple intramuscular and subcutaneous metastases were also uncovered by computed tomography, with some readily palpable on clinical examination. These included deposits within the anterior abdominal wall, right gluteus muscles $(4.5 \mathrm{~cm}$ lesion) and right paraspinal muscle (1.5cm lesion) (Figure 4). Histology from an accessible abdominal wall deposit confirmed a metastatic carcinoma, in keeping with cutaneous involvement by the patient's previous pancreatic adenocarcinoma.

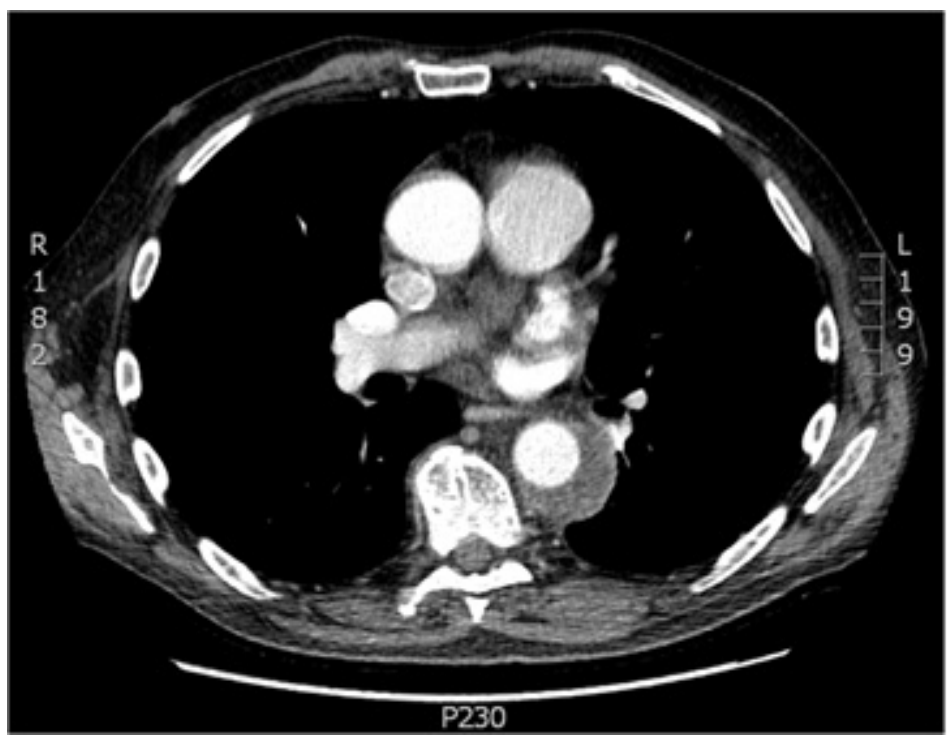

Figure 3: Mediastinal mass encasing descending thoracic aorta, as seen on CT Thorax

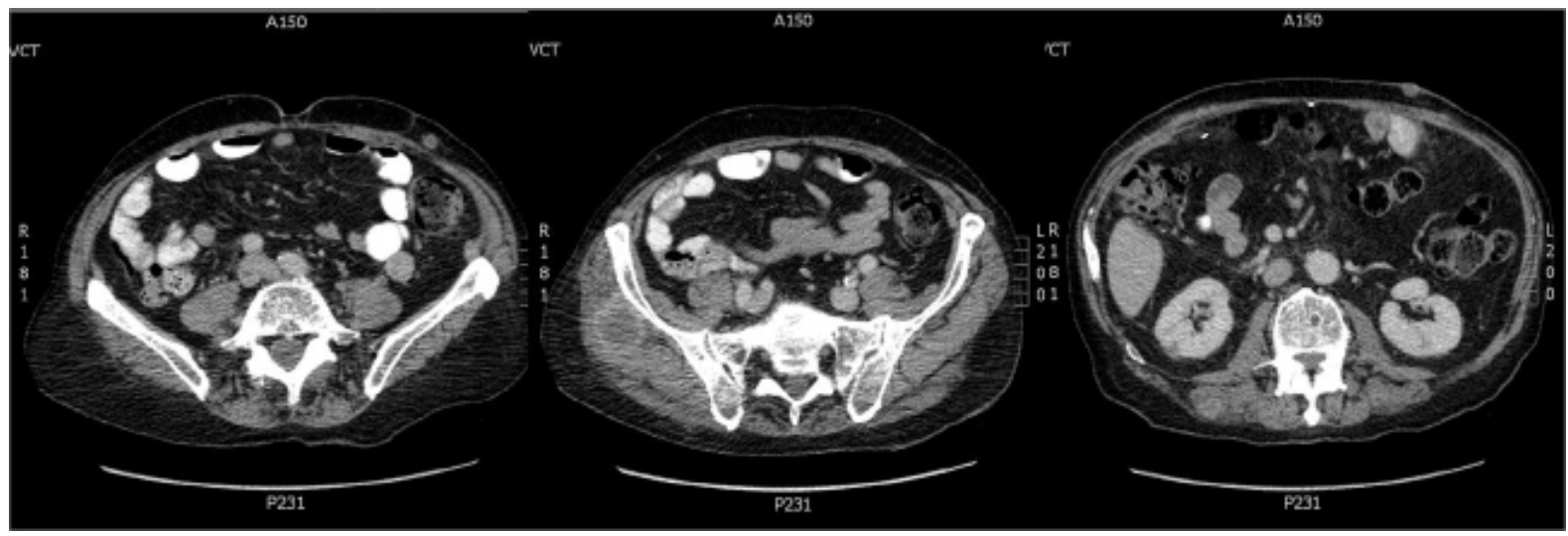

Figure 4: Anterior Abdominal wall deposit, gluteas muscle deposit and right Paraspinal deposit, as seen on CT 
Concurrent multidisciplinary care was instigated for relief of pain and to improve mobility. Input was received from the palliative care team, physiotherapy and occupational therapy departments as well as radiation oncology review.

This clinical scenario was consistent with early diffuse disease recurrence of a pancreatic primary tumour following recent surgical resection of apparent radiologic localized disease. The presenting feature in this case was of symptomatic acrometastases. Initial therapy was with gemcitabine single agent chemotherapy. The presence of acrometastases, together with intramuscular and subcutaneous metastases, is an unusual presentation of pancreatic cancer recurrence and forms the basis for this discussion.

\section{Discussion}

Of all gastrointestinal malignancies, pancreatic is the most common after colorectal [1]. The incidence of pancreatic cancer is estimated at eleven per hundred thousand population in the United States [1]. In Ireland, incidence is similar at about ten per hundred thousand of the population [2]. Pancreatic cancer is difficult to treat and associated with high mortality rates. It is often diagnosed at advanced stages due to a lack of early warning symptoms and signs, and more common in older patients [3,4]. The five year survival for pancreatic cancer ranges from 1.3-8\% and has changed little over years [4-8]. Those patients who are amenable to surgical resection have longer survival rates [7], however recurrence is commonplace.

The 'seed and soil' concept of metastases suggests that bone offers a more suitable environment to promote growth of certain metastases [9]. Primary tumours that metastasise most frequently to bone include prostate, breast and lung [9]. The most common sites for bone metastases, in all patients, are the vertebrae, pelvis, ribs, sternum and skull [10]. Bony metastases below the elbow and the knee are rare. Moreover, metastases to the hand or foot, acrometastasis, are rarer still with an estimated incidence 0.007$0.3 \%$ [10-14]. Hands are more usually involved than feet [10]. Acrometastases have been described in association multiple primary tumours but not pancreatic cancer. A review of metastatic foot lesions revealed genitourinary tumours as the most frequently associated primary [15]. Lung [10,16,17], breast [18,19], renal [11,20], colonic [12,21,22] and, rarely, hepatocellular [23] tumours in association with acrometastatic disease have also been reported.

Pancreatic adenocarcinoma metastases to bone have an overall estimated incidence of between five and twenty percent [24]. Metastatic bone sites include the spine [25], mandible [26] and lower limb [27]. Pancreatic bony metastases have been associated with both osteolytic and osteoblastic lesion formation [28]. To our knowledge there have been no cases of pancreatic tumours in association with distal hand or foot metastatic deposits, as was the presenting feature in our case. The reason why skeletal metastases from pancreatic cancer are uncommon is not clear. It has been postulated that parathyroid hormone-related protein (PTHrP), interleukin 6 (IL-6), vascular endothelial growth factor (VEGF) may play a role as well as other factors such as interleukin 11 (IL-11) and matrix metalloproteinase (MMP).

A further interesting finding in our case is the evidence of multiple intramuscular and subcutaneous metastatic deposits. Gastrointestinal cancers, such as pancreatic, are rarely associated with bone or muscle metastases. Muscular metastases as a feature of pancreatic cancer have been reported in association with other metastatic disease sites [27,29-31], in a similar manner to that of our patient. It has been reported previously that tumour invasion into muscular tissue predicts a relatively worse prognosis and plays an important role in disease progression [32]. There does not appear to be any association between histologic subtype and site or timing of metastatic disease.

Symptoms from bone metastases are rare unless late in the disease course [28]. Treatment options include systemic chemotherapy, radiotherapy and even amputation [33,34]. Gemcitabine and fluorouracil chemotherapy remain the backbone of oncological therapeutics and have modest benefits [5]. There is ongoing research into the most effective ways to utilise these and other cytotoxic agents most effectively [35].

The frequent manifestation of early metastatic disease is testament to the fact that pancreatic cancer, even when localised on scans, is a systemic disease. Occult micrometastases in bone marrow are common and likely explain the poor survival [36,37]. Given that metastatic disease, usually in the peritoneal cavity or liver, is associated with a poor outcome in pancreatic cancer, it may be that life expectancy is not long enough for the micrometastatic disease in bone to manifest itself. The early development of metastatic disease, even in unusual sites, should always be suspected, even in cases of surgery with curative intent.

The use of bisphosphonates or RANK ligand antibodies may provide some benefit in the early treatment of pancreatic cancer given the reported incidence of micrometastatic bone disease. The use of RANK ligand antibodies may prevent chemotaxis between circulating tumour cells and the bone microenvironment [38].

In summary, we describe a first reported case of extensive pancreatic adenocarcinoma with acrometastatic disease. Our patient also developed metastatic deposits in subcutaneous and muscular tissue, both rarely reported with pancreatic cancer. Our case highlights rare sites of metastatic disease secondary to pancreatic cancer and emphasises that pancreas cancer is a systemic disease at diagnosis even when 'localized' on cross sectional imaging. 


\section{References}

1. Shaib YH, Davila JA, El- Serag HB (2006) The epidemiology of pancreatic cancer in the United States: changes below the surface. Aliment Pharmacol Ther 24: 87-94.

2. National cancer registry of Ireland (2012) Cancer Trends - Cancers of the pancreas.

3. Hidalgo M (2010) Pancreatic Cancer. N Engl J Med 362: 1605-17.

4. Porta M, Fabregat X, Malats N, Guarner L, Carrato A, et al. (2005) Exocrine pancreatic cancer: Symptoms at presentation and their relation to tumour site and stage. Clin Transl Oncol 7: 189-97.

5. Sporn JR (1999) Practical recommendations for the management of adenocarcinoma of the pancreas. Drugs 57: 69-79.

6. Pancreatic Cancer Action (2014) UK pancreatic cancer prognosis and survival rates.

7. Sener SF, Fremgen A, Menck HR, Winchester DP (1999) Pancreatic cancer: a report of treatment and survival trends for 100,313 patients diagnosed from 19851995, using the National Cancer Database. J Am Coll Surg 189: 1-7.

8. Gong Z, Holly EA, Bracci PM (2011) Survival in population-based pancreatic cancer patients: San Francisco bay area, 1995-1999. Am J Epidemiol 174: 1373-81.

9. Mundy GR (1997) Mechanisms of bone metastasis. Cancer 80: 1546-56.

10. Lamarca A, Hindi N, Belda-Iniesta C, de Castro J (2012) Foot pain: uncommon presentation of lung cancer. BMJ Case Rep doi: 10.1136/bcr.12.2011.5360.

11. Borgohain B, Borgohain N, Khonglah T, Bareh J (2012) Occult renal cell carcinoma with acrometastasis and ipsilateral juxta-articular knee lesions mimicking acute inflammation. Adv Biomed Res 1: 48.

12. Gharwan H, Yarlagadda L, Duffy A (2012) Acrometastasis as the Initial Presentation of aKRAS-Positive Colon Cancer. Case Rep Oncol 5: 404-8.

13. Anoop TM, George S, Divya KP, Jabbar PK (2010) Metastatic phalangeal osteolysis as an initial presentation of carcinoma colon. Am J Surg 200: e61-3.

14. Sur YJ, Kang YK, Bahk WJ, Chang DK, Rhee SK (2011) Metastatic malignant tumour in the hand. J Plast Surg Hand Surg 45: 90-5.

15. Maheshwari AV, Chiappetta G, Kugler CD, Pitcher JD, Temple HT (2008) Metastatic skeletal disease of the foot: case reports and literature review. Foot Ankle Int 29: 699-710.

16. Campa T, Fagnoni E, Ripamonti C (2004) Palliative Surgery of acrometastases from lung cancer: a case report. Support care cancer 12: 202-4.

17. De Maeseneer M, Machiels F, Naegels S, Verhaeghe W (1995) Hand and foot acrometastases in a patient with bronchial carcinoma. J Belge Radiol 78: 274-5.

18. Biyi A, Oufroukhi Y, Doudouh A (2010) Hand and foot acrometastasis secondary to breast carcinoma. Chir Main 29: 40-3.

19. Wavreille G, Baroncini M, Rtaimate M (2009) A rare cause of pseudoclubbing (pseudohippocratic finger): the acrometastasis. Chir Main 28: 381-3.

20. Choufani E, Diligent J, Galois L, Mainard D (2011) Metastatic renal cell carcinoma presenting as foot metastasis: case report and review of the literature. J Am Podiatr Med Assoc 101: 265-8.

21. Flynn CJ, Danjoux C, Wong J, Christakis M, Rubenstein J, et al. (2008) Two cases of acrometastasis to the hands and review of the literature. Curr Oncol 15: 51-8.

22. Ellington JK, Kneisl JS (2009) Acrometastasis to the foot: three case reports with primary colon cancer. Foot Ankle Spec 2: 140-5.

23. Rauf MS, Motta L, Connolly C (2012) Digital acrometastases as first sign of hepatocellular carcinoma. Scott Med J 57: 247.

24. Borad MJ, Saadati H, Lakshmipathy A, Campbell E, Hopper P, et al. (2009) Skeletal Metastases in Pancreatic Cancer: A retrospective study and review of the literature. Yale J Biol Med 82: 1-6.

25. Lin CT, Tang CT, Liu MY, Ma Hl (2011) Unusual osteoblastic metastases in the spine secondary to adenocarcinoma of the pancreas. Acta Chir Belg 111: 44-5.

26. Jaffa NR, Adam D, Akhtar S, Kyzas PA (2014) Pancreatic adenocarcinoma presenting as mandibular tumor: case report. Oral Surg Oral Med Oral Pathol Oral Radiol 117: 23-6.

27. Chen J, Zheng Q, Yang Z, Huang XY, Yuan Z, et al. (2012) Neuroendocrine carcinoma of the pancreas with soft tissue metastasis. World J Gastroenterol 18: $6682-5$.

28. Pneumaticos SG, Savidou C, Korres DS, Chatziioannou SN (2010) Pancreatic cancer's initial presentation: back pain due to osteoblastic bone metastasis. Eur J Cancer Care 19: 137-40.

29. Chisthi MM, Manju PA (2013) Skeletal muscle metastases from Pancreatic carcinoma - A Case Report and Review of Literature. Indian J Surg 75: 181-4.

30. Wafflart E, Gibaud H, Lerat F, de Kersaint-Gilly A, Leborgne J (1996) Muscular metastasis of cancer of the pancreas. A propos of a case. J Chir (Paris) 133: $167-70$.

31. Iuppa A, Petralia GA, Sciuto A, Romero G (1996) Abdominal wall metastasis of pancreatic adenocarcinoma following laparoscopy. Ann Ital Chir 67: 265-9.

32. Chatterjee D, Rashid A, Wang H, Katz MH, Wolff RA, et al. (2012) Tumor invasion of muscular vessels predicts poor prognosis in patients with pancreatic ductal adenocarcinoma who have received neoadjuvant therapy and pancreaticoduodenectomy. Am J Surg Pathol 36: 552-9.

33. Ibrahim A, Scher N, Williams G, Sridhara R, Li N, et al. (2003) Approval summary for zoledronic acid for treatment of multiple myeloma and cancer bone metastases. Clin Cancer Res 9: 2394-9.

34. Healey JH, Turnbull AD, Miedema B, Lane JM (1986) Acrometastases. A study of twenty-nine patients with osseous involvement of the hands and feet. J Bone Joint Surg Am 68: 743-6.

35. Lockhart AC, Rothenberg ML, Berlin JD (2005) Treatment for pancreatic cancer: current therapy and continued progress. Gastroenterology $128: 1642-54$.

36. van Heek NT, Tascilar M, van Beekveld JL, Drillenburg P, Offerhaus GJ, et al. (2001) Micrometastases in bone marrow of patients with suspected pancreatic and ampullary cancer. Eur J Surg Oncol 27: 740-5.

37. Su D, Yamaguchi K, Tanaka M (2005) The Characteristics of Disseminated Tumor Cells in Pancreatic Cancer: A Black Box Needs to Be Explored. Pancreatology 5: 316-24.

38. Azim H, Azim HA (2013) Targeting RANKL in breast cancer: bone metastasis and beyond. Expert Rev Anticancer Ther 13: 195-201. 


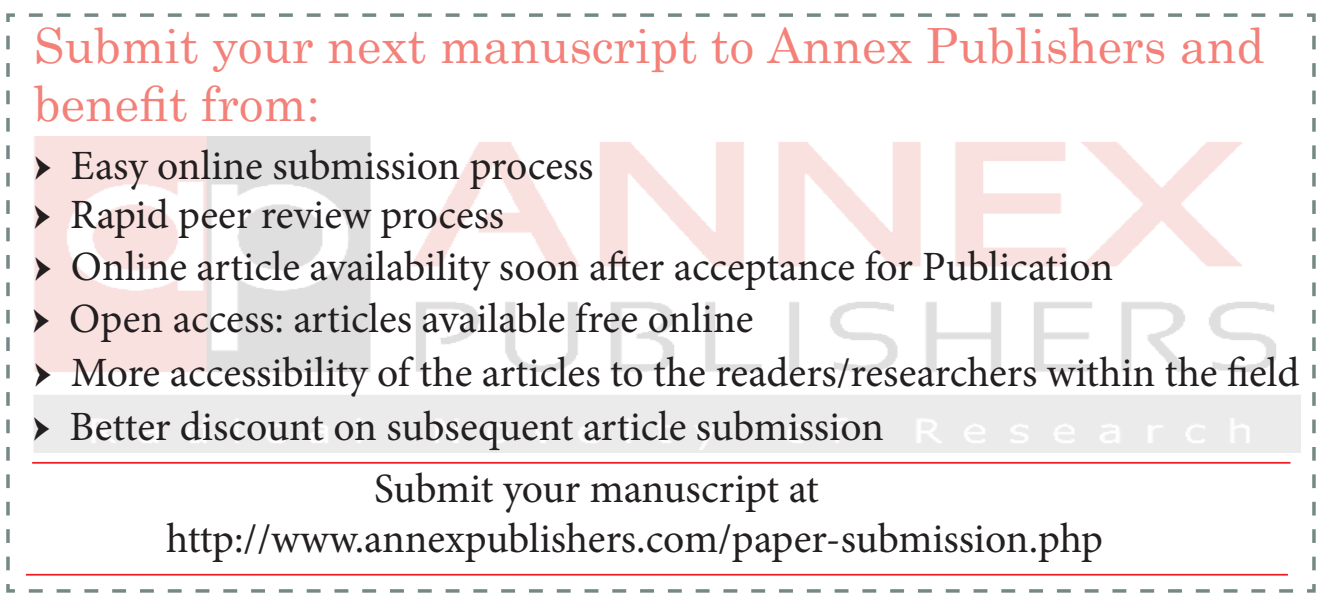

